\title{
The Wnt $/ \beta$-Catenin Asymmetry Pathway Patterns the Atonal Ortholog lin-32 to Diversify Cell Fate in a Caenorhabditis elegans Sensory Lineage
}

\author{
Renee M. Miller and Douglas S. Portman \\ Center for Neural Development and Disease, Department of Biomedical Genetics, University of Rochester School of Medicine and Dentistry, Rochester, New \\ York 14642
}

Each sensory ray of the Caenorhabditis elegans male tail comprises three distinct neuroglial cell types. These three cells descend from a single progenitor, the ray precursor cell, through several rounds of asymmetric division called the ray sublineage. Ray development requires the conserved atonal-family bHLH gene lin-32, which specifies the ray neuroblast and promotes the differentiation of its progeny. However, the mechanisms that allocate specific cell fates among these progeny are unknown. Here we show that the distribution of LIN-32 during the ray sublineage is markedly asymmetric, localizing to anterior daughter cells in two successive cell divisions. The anterior-posterior patterning of LIN-32 expression and of differentiated ray neuroglial fates is brought about by the Wnt/ $\beta$-catenin asymmetry pathway, including the Wnt ligand LIN-44, its receptor LIN-17, and downstream components LIT-1 (NLK), SYS-1 ( $\beta$ catenin), and POP-1 (TCF). LIN-32 asymmetry itself has an important role in patterning ray cell fates, because the failure to silence lin-32 expression in posterior cells disrupts development of this branch of the ray sublineage. Together, our results illustrate a mechanism whereby the regulated function of a proneural-class gene in a single neural lineage can both specify a neural precursor and actively pattern the fates of its progeny. Moreover, they reveal a central role for the $\mathrm{Wnt} / \beta$-catenin asymmetry pathway in patterning neural and glial fates in a simple sensory lineage.

\section{Introduction}

Cell-fate specification in the developing nervous system relies on combinatorial patterns of gene expression determined by both extrinsic and intrinsic developmental cues (Livesey and Cepko, 2001; Bertrand and Hobert, 2010; Hirabayashi and Gotoh, 2010). Although remarkable progress has been made in identifying genetic components important for neural development, our appreciation of the mechanisms that couple cell lineage to the restriction of cell fate is limited. These processes are likely to underlie a variety of developmental disorders of the nervous system; in addition, the success of cell-based therapies will depend on understanding mechanisms that control precursor cell proliferation, cell-fate specification, and differentiation.

The sensory rays of the Caenorhabditis elegans male tail afford the opportunity to dissect a neuroglial cell-fate specification

\footnotetext{
Received Dec. 13, 2010; revised June 20, 2011; accepted July 12, 2011.

Author contributions: R.M.M. and D.S.P. designed research; R.M.M. and D.S.P. performed research; R.M.M. and D.S.P. analyzed data; R.M.M. and D.S.P. wrote the paper.

This work was supported by NIH Grants R01 NS050268 and R01 GM086456 (D.S.P.). Some strains used in this work were provided by the Caenorhabditis Genetics (enter, which is supported by the NIH National Center for Research Resources.We thank Scott Emmons, in whose laboratory lin-17(bx109) was isolated, Shohei Mitani and the National Bioresource Project for providing lin-32 deletion alleles, Scott Clark and Brinda Prasad for lin-32(zd174), David Zarkower and Jennifer Wolff for ezls 10, Andrew Samuelson and Sara Farrell for reagents and advice on RNAi feeding, Massimo Hilliard for the hsp 16.2::lin-44 construct, and Kwi Yeon Lee and Stefani McGregor for expert technical assistance.

The authors declare no competing financial interests.

Correspondence should be addressed to Dr. Douglas S. Portman, Center for Neural Development and Disease, University of Rochester, 601 Elmwood Avenue, Box 645, Rochester, NY 14642. E-mail: douglas.portman@rochester.edu.

DOI:10.1523/JNEUROSCI.6504-10.2011

Copyright $\odot 2011$ the authors $\quad 0270-6474 / 11 / 3113281-11 \$ 15.00 / 0$
}

mechanism with high cellular resolution. The adult male tail bears 18 rays, sensory structures important for mating behavior (Fig. 1A) (Barr and Garcia, 2006). Each ray is an individual sensillum comprising two distinct sensory neurons (RnA and RnB) and an associated glial-like structural cell (Rnst) (Fig. 1B). The generation of exactly one of each of these cell types per ray is ensured by a developmental mechanism in which a single precursor cell, the ray neuroblast Rn.a, undergoes two rounds of cell division to generate one RnA neuron, one RnB neuron, one Rnst cell, and one apoptotic cell (Sulston et al., 1980). This "ray sublineage" is executed synchronously by 18 progenitors during the third and fourth larval stages (Fig. $1 C$ ). Although the patterning of ray cell fates is thought to occur through the segregation of determinants and/or the influence of extracellular signals, these mechanisms remain uncharacterized. Superimposed on these processes common to all rays, diversity among the rays is generated by the Hox genes mab-5 and egl-5 (Chow and Emmons, 1994), the Pax6-related gene mab-18 (Zhang and Emmons, 1995), and a BMP-like signal encoded by $d b l-1$ (Lints and Emmons, 1999; Lints et al., 2004).

The C. elegans atonal ortholog lin-32 is important for the development of several $C$. elegans sensory structures, including the rays, the touch receptor neurons, the deirid (ADE) and postdeirid (PDE) neurons, and the CEPD head sensory neurons (Chalfie and $\mathrm{Au}, 1989$; Zhao and Emmons, 1995; Portman and Emmons, 2000; Doitsidou et al., 2008). In the male tail, lin-32 mutants exhibit significant ray loss; conversely, ectopic lin-32 expression can lead to the development of extra ray-like structures (Zhao 

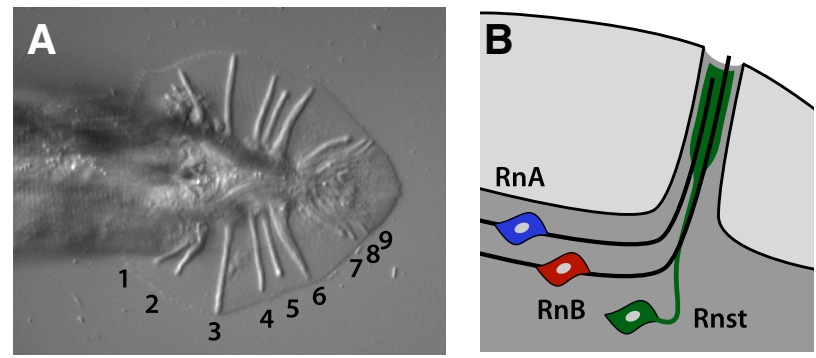

C

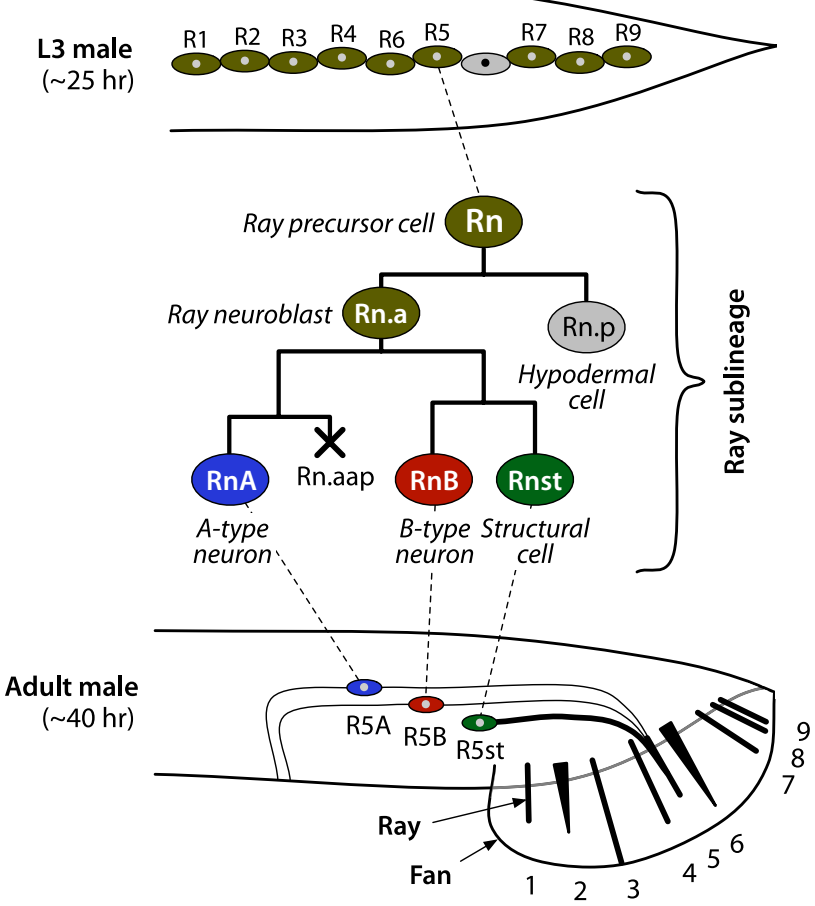

Figure 1. Postembryonic development of the male tail sensory rays. $A$, The adult male tail bears nine pairs of sensory rays. Each ray (numbered 1-9) has characteristic position and morphology. $\boldsymbol{B}$, Each ray is composed of three cell types: two sensory neurons, called RnA and RnB, and an associated glial-like structural cell, Rnst. C, The three cells of each ray are derived through a stereotyped pattern of division and differentiation called the ray sublineage. In the L 3 larval stage, each side of the male tail bears nine ray precursor cells, R1-R9. These cells undergo several rounds of division to generate one hypodermal cell (Rn.p) as well as the three cell types of each mature ray and one apoptotic cell.

and Emmons, 1995). This, together with the observation of lin-32 expression early in the ray sublineage, led to the idea that lin-32 functions as proneural gene for the rays (Zhao and Emmons, 1995). Additional studies indicated that lin-32 has multiple functions in the ray sublineage and that LIN-32 likely acts as a heterodimer with the E47/daughterless-class bHLH protein HLH-2 to activate targets that endow the ray neuroblast with neuroglial potential (Portman and Emmons, 2000, 2004). Here, we find that lin-32 is expressed asymmetrically during the ray sublineage. This asymmetry, as well as the pattern of ray cell-fate specification, is regulated by $\mathrm{Wnt} / \beta$-catenin asymmetry pathway. Our findings lead to an integrated model in which asymmetry cues modulate lin-32 expression to bring about patterning of ray neuroglial cell fates.

\section{Materials and Methods}

C. elegans culture. Standard methods were used for maintenance and construction of C. elegans strains (Brenner, 1974). Mutants used in these studies were as follows: (LG I) lin-17(n671), lin-17(n698), lin-17(bx109), lin44(n1792), unc-13(e51), hlh-2(bx115); (LG II) cwn-1(ok546); (LG IV) egl20(n585), cwn-2(ok895); (LG V) him-5(e1490); (LG X) lin-32(tm1446), lin32(tm2044), lin-32(fs6), lin-32(zd174). Transgenes used were as follows: egIs1 [dat-1::GFP], mhIs9[lin-17::GFP], ezIs10[lin-32::GFP], myIs4[pkd-2::GFP + unc-122:GFP], fsIs14[tba-6::mCherry + tba-9:: YFP + sulp-3::GFP], fsIs18[pkd-2::CFP + trp-4::mCherry + sulp-3::GFP], bxIs14[pkd-2::GFP + pBX1], fsEx148[hsp16.2:LIN-32::GFP + unc122::GFP], fsEx396[hsp16.2::LIN-44 + unc-122::GFP], and qIs74 [POP-1::GFP]. Unless noted otherwise, all strains contained the mutation him-5(e1490) to increase the frequency of males in self-progeny broods.

Scoring of ray phenotypes. Imaging was performed using differential interference contrast (DIC) and epifluorescence optics on a Carl Zeiss Axioplan 2 using Axioskop software. Ray number, an indication of the differentiation of the Rnst cells, was scored by DIC microscopy. To determine whether ray development on the left and right sides can legitimately be considered independent events, we scored ray frequencies on the left and right sides of 46 lin-32(tm2044) males. We found no evidence for correlation between left and right sides $\left(R^{2}=\right.$ 0.017), indicating that, at least for this mutant, each side can indeed be considered to be an independent measurement of ray development. Measurements are therefore reported here in terms of rays per side. Cases in which ray morphology was unusual or fused rays were present are noted in the text.

Ray neuron marker expression was scored and imaged using epifluorescence and the Carl Zeiss Apotome. In some cases (particularly trp-4::mCherry), fluorescent markers did not strongly label neuronal dendrites projecting into the ray. We therefore counted neurons only in males mounted dorsally or ventrally. This allowed unambiguous determination of RnA cell bodies, RnB cell bodies, and Rnst (as judged by the presence of the ray process itself by DIC).

lin-17(bx109) genetic mapping. bx109 is a recessive allele that was isolated in an ethyl methanesulfonate (EMS) screen for males with ray-loss defects (Portman and Emmons, 2000). Two- and three-factor mapping crosses (data not shown) localized the mutation to the left arm of chromosome I. The failure of $b \times 109$ to complement the ray-loss phenotype of lin-17(n698) indicated that $b \times 109$ was likely to be a lin-17 allele. Sequencing of the lin-17 locus in $b \times 109$ mutants revealed a G-to-A mutation at position 5 of the third intron. This mutation can disrupt splicing of the lin-17 transcript (data not shown) and may therefore decrease the level of functional LIN-17 protein.

Inducible expression of lin-32 and lin-44. A construct containing the lin-32 cDNA fused to GFP driven by the hsp16-2 promoter (Fire Lab Vector kit; Addgene) was generated using standard methods and used to produce the extrachromosomal array $f_{s} E x 148$. To induce expression from the transgene, synchronized L3 animals (representing a collection of individuals at different stages early in the ray sublineage) harboring $f_{s}$ Ex 148 were heat shocked with a 20 min incubation in a $33^{\circ} \mathrm{C}$ water bath, followed by recovery at $15^{\circ} \mathrm{C}$ for $24-48 \mathrm{~h}$. This duration of heat shock rapidly induced GFP expression in all somatic cells, including the dividing cells of the ray sublineage (data not shown). hsp-16.2::LIN-32::GFP expression was typically extinguished $6-8 \mathrm{~h}$ after heat shock. After reaching adulthood, males were examined for the presence and appearance of rays (RnSt cells), RnA neurons (using DAT-1::GFP expression), and RnB (using PKD-2::GFP expression). Similar protocols were followed to induce LIN-44 expression. A plasmid containing the $h s p 16.2$ promoter driving lin-44 coding sequence was generously provided by Massimo Hilliard (Queensland Brain Institute, University of Queensland, Brisbane, QLD, Australia) and used to produce the extrachromosomal array $f_{s} E x 396$. To induce LIN-44 expression ectopically, L3 males were exposed to a $33^{\circ} \mathrm{C}$ heat shock for $30 \mathrm{~min}$. Once these males reached adulthood, they were examined, and the presence and appearance of rays, $\mathrm{RnA}$, and $\mathrm{RnB}$ neurons was quantified using the integrated $f_{s} I s 18$ transgene.

RNA interference. Synchronized cultures of L1 animals carrying the $f_{\text {SIs } 14}$ or $f_{\text {SIs }} 18$ transgenes were generated by exposing gravid hermaphrodites to an $\mathrm{NaOH}$ bleach solution (50\% $1 \mathrm{M} \mathrm{NaOH}, 17 \%$ Clorox bleach) and recovering the embryos in M9 for $48 \mathrm{~h}$ with gentle shaking. L1 worms were placed on agar plates containing isopropyl- $\beta$-D- 
A

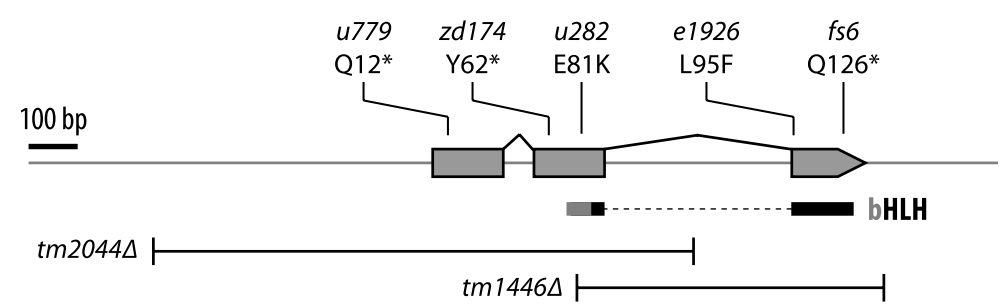

B
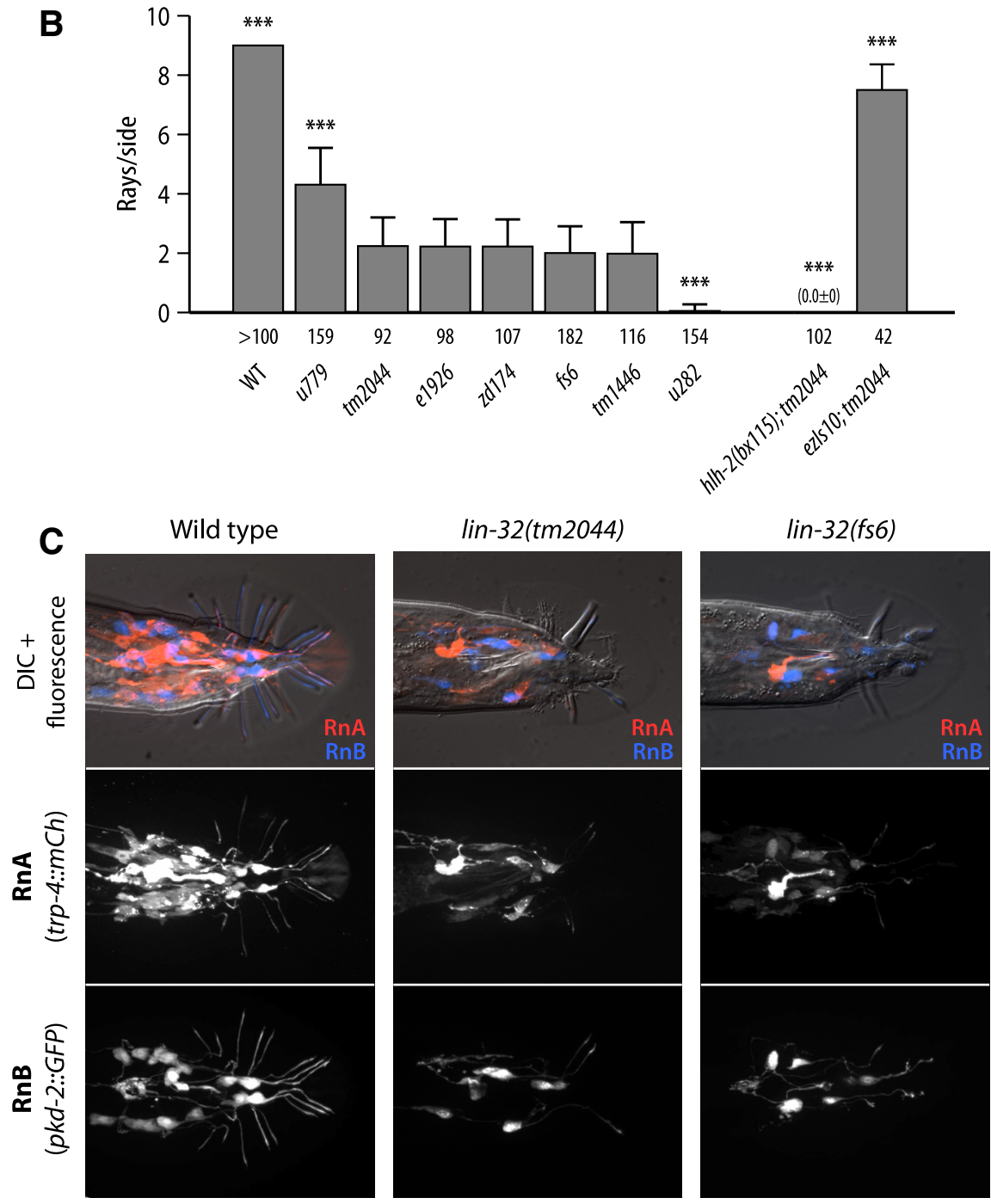

Figure 2. Loss of lin-32 function significantly disrupts but does not abolish ray development. $\boldsymbol{A}$, The structure of the lin-32 locus, with point mutations and deletion alleles indicated. The portion of the lin-32 ORF that encodes the bHLH domain is shown underneath the lin-32 exon-intron structure. $\boldsymbol{B}$, Ray frequency, in rays per side, in wild-type males as well as lin-32 mutants. Asterisks indicate statistical significance compared with lin-32(tm2044), as assessed by one-way ANOVA and Dunnett's multiple comparison test. ${ }^{* *} p<0.001$. Figures below each bar indicate the number of sides scored. $\boldsymbol{C}$, Wild-type males have nine rays per side. Most of the ray RnA neurons express the marker trp-4, whereas eight of the nine RnB neuron pairs express the marker $p k d-2$. In lin-32(tm2044) and lin-32(fs6) mutants, stochastic but generally severe ray loss is observed, as seen in both the ray process itself (which is generated by the Rnst ray structural cell) and the expression of RnA and RnB markers.

thiogalactopyranoside seeded $24 \mathrm{~h}$ previously with bacteria expressing double-stranded RNA directed to pop-1, sys-1, or lit-1 or the empty vector L4440. After $3 \mathrm{~d}$, adult male worms were scored for the presence of $\mathrm{RnA}, \mathrm{RnB}$, and Rnst cells using marker gene expression and DIC imaging. RNAi efficacy was confirmed by the almost completely penetrant sterility and protruding/bursting vulva phenotypes of adult hermaphrodites grown on pop-1, sys-1, and lit-1 RNAi clones.

\section{Results}

lin-32 is important but not essential for ray development

Previous work has found that lin-32 mutants have severe defects in ray development (Zhao and Emmons, 1995; Portman and Emmons, 2000). These mutants fall into three categories with respect to their ray phenotypes (Fig. $2 A, B$ ). The most severe allele, lin-32(u282), causes virtually complete ray loss of all nine rays (Zhao and Emmons, 1995), whereas the least severe, lin-32(u779), results in only modest ray loss (approximately four rays per side develop in these males) (Portman and Emmons, 2000). The intermediate class, represented by lin-32(e1926) and lin32(bx46) (Zhao and Emmons, 1995), exhibits moderate ray loss, with mutant males exhibiting a mean ray frequency of 2.1 rays per side. Both the weak and intermediate phenotypes are characterized by a strong bias in the pattern of ray loss: rays $1,2,7$, and 8 almost never form in these mutants, whereas rays 5, 6, and 9 are observed relatively frequently (Zhao, 1995; Zhao and Emmons, 1995; Portman and Emmons, 2000). This is thought to result from differences in the level of activation of lin-32 and its heterodimerization partner hlh-2, and possibly other ray-promoting genes, by the Hox genes mab-5 and egl-5 (Chow and Emmons, 1994; Zhao and Emmons, 1995; Ferreira et al., 1999; Portman and Emmons, 2000; Teng et al., 2004; Ross et al., 2005).

Although the X-linkage of lin-32 complicates analysis in males, genetic data had suggested that all known alleles were hypomorphs and that the lin-32 null phenotype was zygotic lethality (Zhao and Emmons, 1995). However, the recent isolation of deletion alleles removing large regions of lin-32 (Fig. 2A) unexpectedly revealed that lin-32 null animals are viable and fertile. lin$32(\mathrm{tm} 1446)$ is a $511 \mathrm{bp}$ deletion that removes essentially all coding sequence for the bHLH domain. lin-32(tm2044) is a larger deletion that removes two of three exons and 568 bp of upstream sequence, replacing it with an insertion of $6 \mathrm{bp}$. Both of these alleles are thus likely to be true nulls. lin32(tm1446) and lin-32(tm2044) homozygous hermaphrodites were viable and fertile. Furthermore, mutant males did not completely lack rays; rather, the phenotypes of these alleles were intermediate in the lin-32 allelic series, similar to e1926 (Fig. 2B). Two newly isolated EMS alleles, lin-32(fs6) and lin32(zd174) (generously provided by B. Prasad and S. Clark, University of Nevada, Reno, NV), also had e1926-like phenotypes (Fig. $2 A, B)$. Among this group of intermediate-strength alleles, none showed significant differences in ray frequency from lin- 
Table 1. Ray development defects in lin-32 and Wnt/ $\beta$-catenin asymmtery mutants ${ }^{a}$

\begin{tabular}{|c|c|c|c|c|}
\hline Genotype $^{b}$ & Rays per side & $\begin{array}{l}\text { RnAs (trp-4::mCh) } \\
\text { per side }\end{array}$ & $\begin{array}{l}\text { RnBs (pkd-2::GFP) } \\
\text { per side }\end{array}$ & $\begin{array}{l}n \\
\text { (sides) }\end{array}$ \\
\hline Wild-type & $9.0 \pm 0.3$ & $6.2 \pm 1.5$ & $7.9 \pm 0.5$ & 64 \\
\hline $\operatorname{lin}-32(\operatorname{tm} 2044 \Delta)$ & $2.3 \pm 1.0^{* * *}$ & $2.0 \pm 1.0^{* * *}$ & $3.3 \pm 1.0^{* * *}$ & 88 \\
\hline lin-17(n671) & $0.0^{* * *}$ & n.d. & n.d. & 18 \\
\hline lin-17(n698) & $3.7 \pm 2.5^{* * *}$ & $7.2 \pm 3.4^{* *}$ & $5.6 \pm 3.5^{* * *}$ & 110 \\
\hline lin-17(bx109) & $6.8 \pm 2.5^{* * *}$ & $6.7 \pm 2.3$ & $7.2 \pm 3.0$ & 78 \\
\hline lin-44(n1792) & $4.5 \pm 2.0$ & $4.0 \pm 2.4$ & $7.2 \pm 1.6$ & 36 \\
\hline $\begin{array}{l}\text { lin-44(n1792); } \\
\text { egl-20(n585) }\end{array}$ & $3.2 \pm 2.0^{\wedge}$ & $5.5 \pm 1.6^{\wedge}$ & $7.3 \pm 1.2$ & 38 \\
\hline $\begin{array}{l}\text { lin-44(n1792); } \\
\quad \text { cwn-1; cwn-2 }\end{array}$ & $3.6 \pm 2.1$ & $5.2 \pm 1.1^{\wedge}$ & $7.4 \pm 1.6$ & 32 \\
\hline Control RNAi & $8.9 \pm 0.4$ & n.d. & n.d. & 58 \\
\hline lit-1(RNAi) & $6.6 \pm 1.7^{\mathrm{tt \dagger}}$ & n.d. & n.d. & 93 \\
\hline sys-1(RNAi) & $8.1 \pm 1.1^{\mathrm{ttt}}$ & n.d. & n.d. & 94 \\
\hline pop-1(RNAi) ${ }^{c}$ & $3.9 \pm 2.3^{t+t}$ & n.d. & n.d. & 100 \\
\hline
\end{tabular}

n.d., Not determined. ${ }^{* *} p<0.01,{ }^{* * *} p<0.001$ compared with wild-type controls. ${ }^{*} p<0.05,{ }^{* *} p<0.01$ compared with lin-44(n1792). ${ }^{\text {t+t }} p<0.001$ compared with control RNAi.

${ }^{a}$ All values are reported as mean $\pm S D$.

${ }^{b}$ All strains contain him-5(e1490) (see Materials and Methods). Except for the RNAi experiments, strains listed here also contained the transgene fs/s 18 .

"Many pop-1(RNAi) animals had large "fused" rays that could arise from either the fusion of two separate rays (as a result of a defect in ray identity specification) or the development of multiple Rnst ray structural cells from a single ray sublineage.

32(tm2044). Additionally, all shared the same bias in ray frequencies originally observed in lin-32(e1926) (Zhao and Emmons, 1995), such that rays 5, 6, and 9 were most commonly observed (data not shown).

Together, these results demonstrate that lin-32 is important but not essential for ray development, implying the existence of other factor(s) that can promote ray development in lin-32 mutants. A strong candidate for such a factor is HLH-2, the C. elegans E47/ daughterless ortholog (Krause et al., 1997). Previous work has demonstrated that HLH-2 can heterodimerize with LIN-32 in vitro and that weak $h l h-2$ mutations enhance ray loss in lin-32 mutants (Portman and Emmons, 2000). We found that $h l h-2(b \times 115)$ also enhanced the ray-loss phenotype of lin-32(tm2044), such that essentially no ray development is observed in hlh-2(bx115); lin32(tm2044) males (Fig. 2 B). This suggests that, at least in the absence of lin-32 function, HLH-2 itself can promote ray development, as either a homodimer or a heterodimer with an unidentified partner. Nevertheless, during normal ray development, HLH-2 likely acts primarily as the heterodimerization partner for LIN-32.

In lin-32(e1926) males, the entire ray sublineage often fails because of a transformation of the ray neuroblast to a hypodermal cell (Zhao and Emmons, 1995). However, in the same individual, other ray neuroblasts can generate partial ray sublineages in which only one or two of the three cell types that comprise each ray differentiate normally (Portman and Emmons, 2000). We used lin-32(tm2044) to confirm that this "uncoupling" of ray cell-fate specification could be observed in animals carrying the bona fide null allele. Using Ptrp-4::mCherry, a marker for most RnA neurons (Hurd et al., 2010), and Ppkd-2::CFP, a marker for all RnB neurons but R6B (Barr et al., 2001), we found a marked decrease in the number of labeled RnA and RnB neurons in lin32(tm2044) mutants compared with wild-type (Table 1, Fig. 2C). In many cases, all three ray cell types of a given ray were missing. We also observed many instances in which partial ray sublineages were generated, i.e., we could detect well-formed rays lacking $\mathrm{RnA}$ or RnB dendrites, as well as the converse, ray neurons without cognate rays (Fig. $2 C$ ). Thus, in the absence of $l i n-32$, stochastic failures in the development of each of the three ray cell types can occur independently, highlighting the importance of independent regulatory events that promote multiple steps of ray development.

lin-32 is expressed asymmetrically during the ray sublineage Previous work has shown that lin-32::lacZ and lin-32::GFP reporter genes are expressed during the ray sublineage (Zhao and Emmons, 1995; Portman and Emmons, 2000). To better understand the role of lin-32 in ray development, we more carefully examined its expression using the LIN-32::GFP translational reporter transgene ezIs10 (Ross et al., 2005). This transgene nearly completely rescued the ray loss of lin-32(tm2044) males (Fig. 2 B), indicating that it encodes a functional protein.

In contrast to a previous report (Zhao and Emmons, 1995), we did not observe LIN-32::GFP expression in the Rn ("ray precursor") cells. Instead, expression was first apparent in the anterior daughters of these cells, the Rn.a or "ray neuroblast" cells (Fig. 3Ai). In wild-type animals, these cells undergo two additional rounds of division to generate the four postmitotic ray cells (Fig. 3B). Unexpectedly, we found that LIN-32::GFP was asymmetrically distributed in these divisions. When Rn.a divides, LIN-32::GFP is initially detectable in both daughter cells (Fig. 3 Aii). However, its expression is maintained only in the anterior daughter, Rn.aa, such that LIN-32::GFP becomes rapidly undetectable in its sister Rn.ap (Fig. 3Aiii). This pattern is repeated in the division of Rn.aa: shortly after an initially similar distribution of LIN-32::GFP (Fig. 3Aiv), Rn.aaa displays high LIN-32::GFP levels, whereas expression in its sister Rn.aap (Fig. $3 A v$ ), fated to undergo programmed cell death, is weaker. Several hours later, LIN-32::GFP becomes undetectable in Rn.aaa itself, as this cell differentiates into an RnA neuron. Thus, LIN-32 expression becomes reiteratively restricted to the anterior daughter cell shortly after two successive cell divisions. This patterned expression raised the possibility that LIN-32 may itself have a role in patterning cell fates during the ray sublineage.

\section{lin-17/Frizzled signaling promotes ray development}

Polarity in the ray sublineage cell divisions and in LIN-32 asymmetry is oriented along the anterior-posterior (A-P) body axis. The five C. elegans Wnt ligands have been proposed to provide a source of global positioning information along this axis to regulate cell division, migration, and fate (Eisenmann, 2005). Indeed, previous work has pointed to a role for Wnt signaling in regulating ray development: the Frizzled-family receptor LIN-17 was found to be expressed during the ray sublineage (Sawa et al., 1996), and males lacking the Wnt ligand LIN-44 appeared to have polarity reversals in the ray sublineage (Herman and Horvitz, 1994).

In a previous genetic screen for ray development mutants (Portman and Emmons, 2000), we isolated a new lin-17 allele, lin-17(bx109). This allele disrupts lin-17 splicing (see Materials and Methods) and appears to be a weak hypomorph. Consistent with this, these males have mild ray loss (6.8 rays per side, $n=78$ ) (Table 1) and occasionally show duplication of the T.a lineage, resulting in an extra set of T-derived rays; lin-17(bx109) hermaphrodites occasionally exhibit an ectopic pseudovulva (the "Biv" phenotype) (data not shown). We also examined males carrying stronger lin-17 alleles. Ray phenotypes are difficult to assess in animals homozygous for the putative null allele lin17(n671): by late larval development, most of these males are grossly deformed and have necrotic tails. However, rare animals that do survive to adulthood and have tails whose morphology is interpretable appear to completely lack rays ( 0 rays per side, $n=$ 

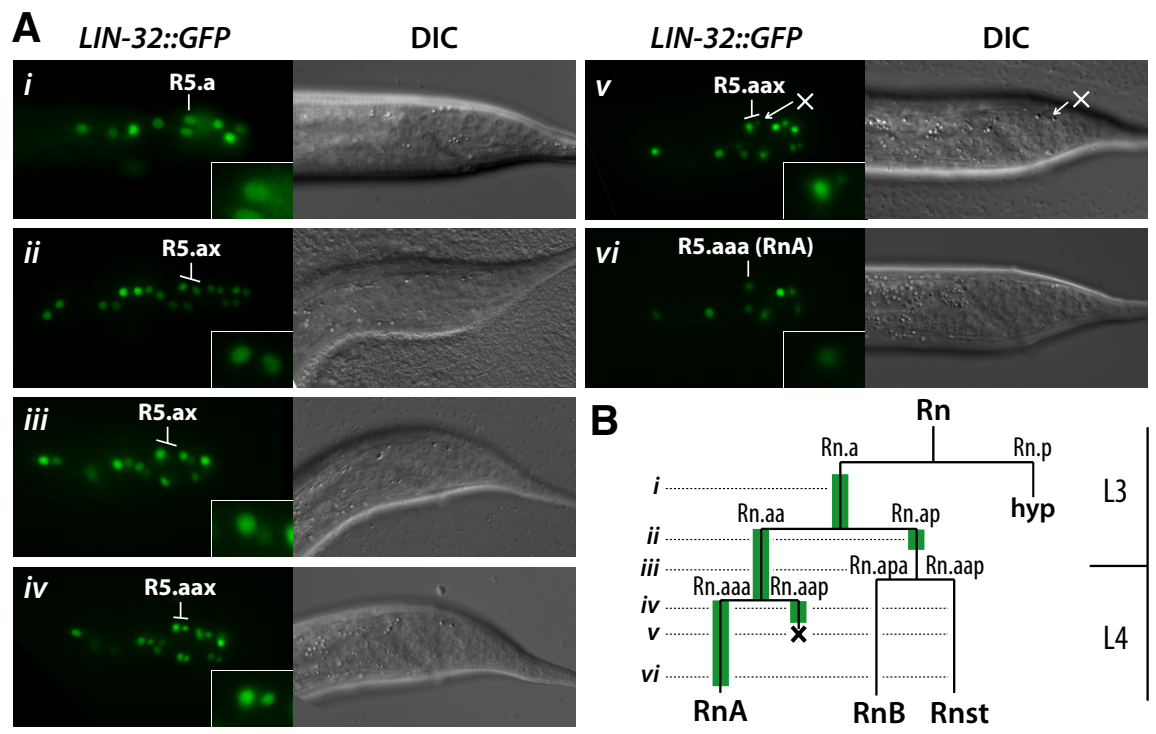

Figure 3. $\quad l i n-32$ is expressed asymmetrically during ray development. $A$, An individual male followed from late $L 3$ to early $L 4$, $\sim 10$ h. Expression of LIN-32::GFP is shown on the left of each panel. The R5 (ray 5) lineage is indicated in each panel and enlarged in the bottom right corner of the fluorescence image. The right side of each panel shows a DIC image of the same animal shortly after the fluorescence image was captured. $\boldsymbol{B}$, The ray sublineage, indicating the asymmetric expression of LIN-32::GFP with green vertical lines. $\boldsymbol{i}-\boldsymbol{v} \boldsymbol{i}$ indicate the six time points shown in $\boldsymbol{A}$.

18). Males bearing the hypomorphic allele lin-17(n698) are much healthier and exhibit a less severe ray defect (3.7 rays per side, $n=$ 110) (Table 1). Together, these results indicate that lin-17 function is necessary for proper ray development.

\section{The Wnt/ $\beta$-catenin asymmetry pathway regulates the specification of ray cell fates}

In C. elegans, a canonical Wnt signaling pathway uses the $\beta$-catenin BAR-1 to regulate several events, including vulval and Q neuroblast development (Korswagen et al., 2000). A second $\beta$-catenin-dependent mechanism called the "Wnt/ $\beta$-catenin asymmetry pathway" (Mizumoto and Sawa, 2007; Phillips and Kimble, 2009) has broader roles during C. elegans development. This mechanism has been repeatedly implicated in generating anterior-posterior polarity, acting in the division of the embryonic blastomere EMS, the tail hypodermal cell T, the somatic gonad precursors $\mathrm{Z} 1$ and $\mathrm{Z} 4$, and in the stem cell-like divisions of the lateral hypodermal seam cells (Mizumoto and Sawa, 2007; Phillips and Kimble, 2009). It has also been found recently to have an important role in cell-fate specification during embryonic nervous system development (Bertrand and Hobert, 2009). A key characteristic of this pathway is its use of two $\beta$-catenins, WRM- 1 and the divergent $\beta$-catenin SYS-1. In the Wnt $\beta$ catenin asymmetry pathway, receptor activation (generally on the posterior aspect of a dividing cell) leads to the asymmetric segregation of key pathway components, including LIN-17/Fz and DSH-2/Dsh (generally in the posterior daughter) and WRM$1 / \beta$-catenin, LIT-1/NLK, APR-1/APC, and PRY-1/Axin (generally in the anterior daughter) (Mizumoto and Sawa, 2007; Phillips and Kimble, 2009). These asymmetries trigger differences in the balance of two key effectors, POP-1/TCF and SYS-1/ $\beta$-catenin, in the two daughter nuclei. In the anterior nucleus, a low SYS-1/POP-1 ratio allows POP-1 to repress its transcriptional targets; in the posterior cell, a high SYS-1/POP-1 ratio allows activation of targets by a SYS-1-POP-1 complex (Kidd et al., 2005; Phillips et al., 2007). Disruption of the function of components of the $\mathrm{Wnt} / \beta$-catenin asymmetry pathway often leads to the adoption of anterior "default" fates by both daughter cells (Mizumoto and Sawa, 2007; Phillips and Kimble, 2009). Consistent with a role for this mechanism in patterning the ray sublineage, we found that the expression of POP-1 was highly asymmetric in these cells (Fig. 4A). Anterior cells exhibited consistently higher levels of POP-1::GFP than their posterior sisters, reminiscent of observations in other lineages regulated by the $\mathrm{Wnt} / \beta$-catenin asymmetry pathway (Mizumoto and Sawa, 2007; Phillips and Kimble, 2009).

These findings indicated that the Wnt/ $\beta$-catenin asymmetry pathway might have a functional role in patterning anterior-posterior asymmetry during ray development. Addressing this issue is made somewhat more challenging because each ray sublineage comprises three distinct divisions that could be patterned by Wnt signaling, Rn.a[a/p] (anterior branch vs posterior branch), Rn.aa[a/p] (RnA neuron vs apoptotic cell), and Rn.ap $[\mathrm{a} / \mathrm{p}]$ (RnB neuron vs Rnst glial cell) (Fig. 4C). As such, hypomorphic alleles or RNAi knockdown could cause a variety of incompletely penetrant cellfate specification defects. Moreover, the Wnt $\beta$-catenin asymmetry pathway also has earlier roles in patterning in the V5, V6, and $\mathrm{T}$ seam-cell lineages from which the ray precursor cells are derived (Sulston et al., 1980; Banerjee et al., 2010; Gleason and Eisenmann, 2010; Ren and Zhang, 2010). In general, however, the loss of positive-acting components of this pathway might be expected to transform cell fates away from more posterior ray sublineage fates ( $\mathrm{RnB}$ and $\mathrm{Rnst}$ ) toward the anteriormost ( $\mathrm{RnA})$ fate (Fig. 4C).

Supporting this idea, we observed a variety of phenotypes in lin-17 mutants. In males carrying the strong allele lin-17(n671), RnB marker Ppkd-2::CFP expression was detectable in few cells (Fig. $4 E$ ); in contrast, numerous cells expressing the RnA marker Ptrp-4::mCherry were sometimes apparent. However, gross disorganization, necrosis, and weak marker expression in these animals prevented accurate counting of cell numbers. With the weaker alleles lin-17(n698) and lin-17(bx109), we observed slight increases in Ptrp-4::mCherry-expressing RnA neurons compared with wild-type males (Fig. $4 D, E$ ). Simultaneously, the numbers of $P$ pkd-2::CFP-expressing RnB neurons were reduced, although not as severely as were the number of Rnst cells, as judged by the loss of rays (Fig. $4 D, E$ ). We verified the increase in RnA-like neurons using a second marker, Pdat-1::GFP, which is expressed in three RnA neurons per side $(\mathrm{R}[5,7,9] \mathrm{A})$ in wild-type males (Nass et al., 2002; Lints et al., 2004). Again, lin-17 mutants possessed extra RnA-like cells: we observed 8.4, 7.0, and 4.5 GFP-positive cells per side in lin-17(n671), lin-17(n698), and lin-17(bx109) males, respectively $(n=16,30$, and 19 sides, respectively) (Fig. 4D,F). Although we cannot rule out the possibility that these extra GFP-positive cells result from the adoption of dopaminergic fates from rays other than 5, 7, and 9, their appearance in small discrete groups makes it more likely that they result from transformations of more posterior cells in the ray 5, 7, and 9 sublineages to the $\mathrm{R}[5,7,9] \mathrm{A}$ fate. Together, these data indicate that multiple ray cell-fate transformations can occur in lin-17 mutants, with a general bias toward an increase in the 
adoption of the anteriormost ray cell fate $(\mathrm{RnA})$ at the expense of the posteriormost (Rnst).

Our finding that $\operatorname{lin}-17(n 671)$ males exhibit incomplete posterior-to-anterior cell-fate transformations (Fig. $4 E, F$ ) is reminiscent of results on the asymmetry of seam cell divisions. Although these earlier seam cell asymmetric divisions rely on components of the $\mathrm{Wnt} / \beta$-catenin asymmetry pathway, they depend only partially on lin-17 and are thought to be essentially independent of Wnt ligands (Banerjee et al., 2010; Gleason and Eisenmann, 2010; Ren and Zhang, 2010), suggesting that both extrinsic and intrinsic polarity cues can act via the $\mathrm{Wnt} / \beta$-catenin asymmetry pathway to generate asymmetry in cell fate. Three other results indicated that the generation of A-P asymmetry in the ray sublineage might also be partially independent of extrinsic cues. First, we failed to observe any anterior-posterior asymmetry in the distribution of LIN-17::GFP during the ray sublineage (Fig. $4 B$ ). However, any subtle asymmetry in LIN-17 localization or abundance would not likely be detectible with this approach. Second, we found that the ray sublineage only partially requires Wnt ligand activity. Of the five C. elegans Wnt ligands, three (LIN-44, CWN-1, and CWN-2) are expressed in the tail tip (Gleason et al., 2006). We found that lin-44(n1792) null males showed mild but significant ray loss, which was slightly enhanced by $c w n-1$; cwn-2 or by egl-20, a fourth Wnt ligand expressed anterior to the rays (Table 1). Unexpectedly, loss of these other Wnt ligands slightly suppressed the RnA defects of lin-44 mutants. Third, we asked whether LIN-44 might provide an instructive polarity cue by determining whether ectopically expressed LIN-44 could cause anterior-to-posterior cell-fate transformations. Although broad expression of LIN-44 from a heat-shock promoter during late $\mathrm{L} 3$ did cause minor alterations in ray position and morphology, it failed to bring about detectable changes in cell fate (data not shown), although it is possible that the timing or strength of LIN-44 expression was not optimal. Together, these results indicate that asymmetric divisions in the ray sublineage, like those in the seam cells, may be only partially dependent on extrinsic polarity information. Alternatively, other Wnt ligands and receptors (Eisenmann, 2005) might act together with LIN-44 and LIN-17 to bring about robust anterior-posterior patterning during ray development.

To ask whether other components of the $\mathrm{Wnt} / \beta$-catenin asymmetry pathway are important for ray sublineage patterning, we performed RNA interference against the Nemo-like kinase lit- 1 , the divergent $\beta$-catenin sys-1, and the TCF transcription factor pop-1. To bypass embryonic requirements for these genes, we fed newly hatched L1 larvae with Escherichia coli expressing
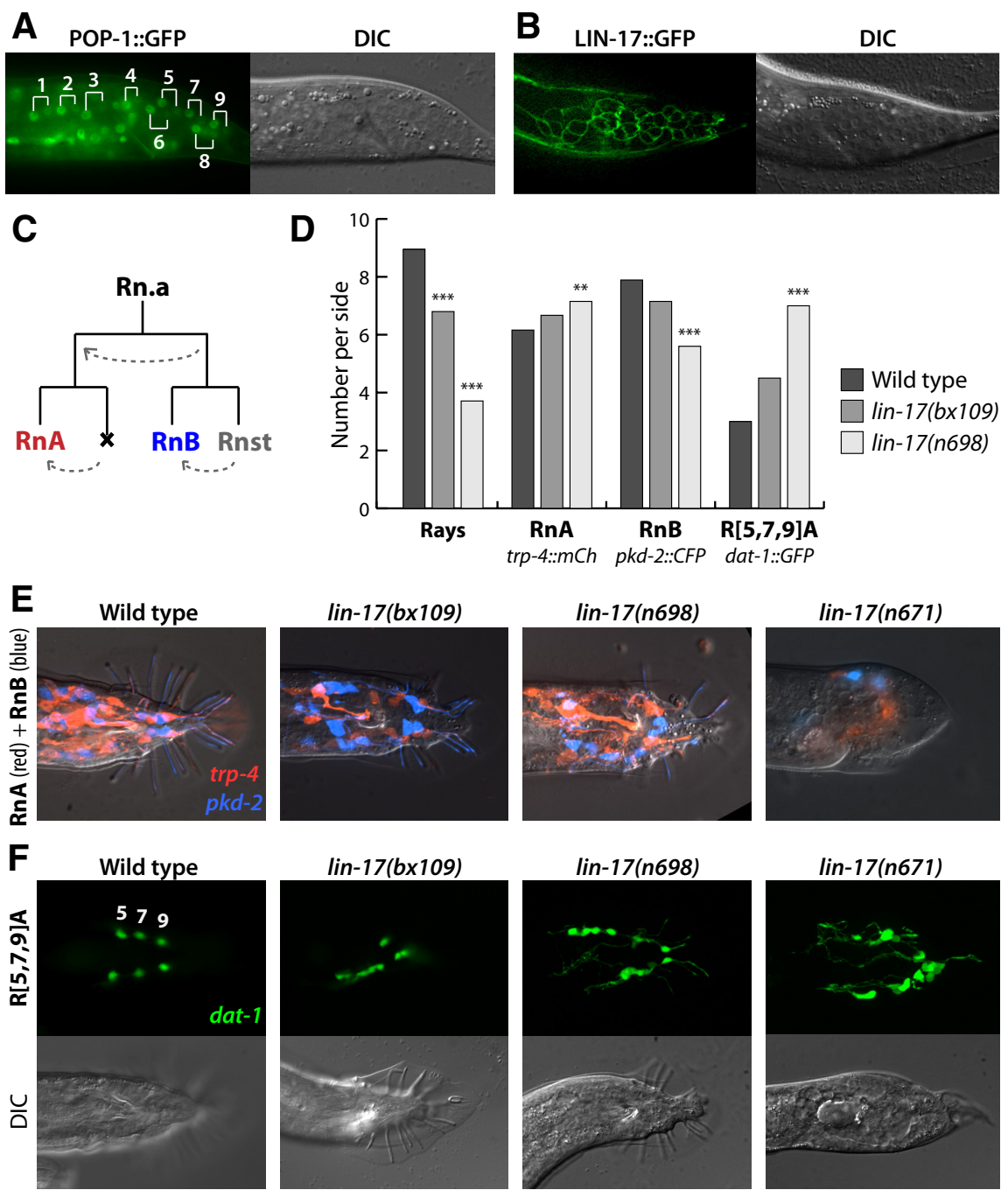

Figure 4. lin-17 regulates the patterning of the ray sublineage. $A$, POP-1::GFP is expressed asymmetrically during ray development. Shown is an Rn.aa-stage animal; each Rn.aa/ap pair is indicated with a bracket. $\boldsymbol{B}$, The Wnt receptor LIN-17 is widely background. An Rn.aa-stage animal is shown; LIN-17::GFP fluorescence is shown above the corresponding DIC image. C, Three terior-posterior asymmetries in the ray sublineage could be regulated by Wnt signaling. Dashed arrows indicate possible . rmations in lin-17 mutants. $\boldsymbol{D}$. The frequency of rays, $\mathrm{RnA}$ and $\mathrm{RnB}$ neurons in wild-type and lin-17 mutant males. - 4 and pkd-2 expression marks most $\mathrm{RnA}$ and $\mathrm{RnB}$ neurons, respectively. dat- 1 expression marks the RnA neurons of rays 5, 7 Asterisks indicate statistical significance compared with wild type, as assessed by one-way ANOVA and $t$ test with Bonferroni's correction: ${ }^{* *} p<0.01 ;{ }^{* * *} p<0.001$. $\boldsymbol{E}, \boldsymbol{F}$, Adult male tails in wild-type and lin-17 mutants carrying trp-4 and $p k d-2$ markers $(\boldsymbol{E})$ or a dat-1 marker $(\boldsymbol{F})$ to visualize ray neurons.

dsRNA against these targets. Each of these treatments resulted in ray loss (Table 1). lit-1(RNAi) and sys-1(RNAi) ray loss was modest but highly statistically significant (in both cases, $p<0.001$ compared with control RNAi); the low penetrance of these effects may stem from incomplete knockdown, a known property of the RNAi feeding technique. In contrast, pop-1(RNAi) led to more severe defects. Although many of these animals had grossly deformed tails in which ray development could not be reliably assessed, others showed extensive ray defects (Fig. 5A). Interestingly, we observed two phenotypes consistent with loss of normal anterior-posterior specification. First, some pop-1(RNAi) males showed extensive ray loss and ectopic expression of the $\mathrm{RnA}$ marker tba-9::YFP (Fig. 5A), consistent with posterior-to-anterior fate transformations seen on pop-1(RNAi) in the T lineage (Herman, 2001) and with distal-to-proximal transformations observed in the somatic gonad (Siegfried and Kimble, 2002). We 


\section{pop-1(RNAi)}

A
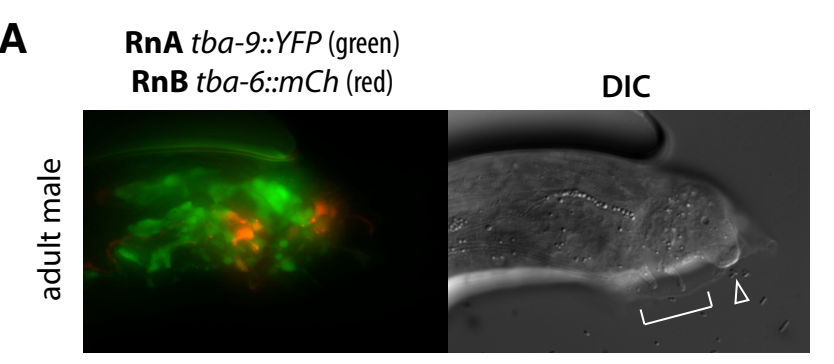

B

LIN-32::GFP

DIC

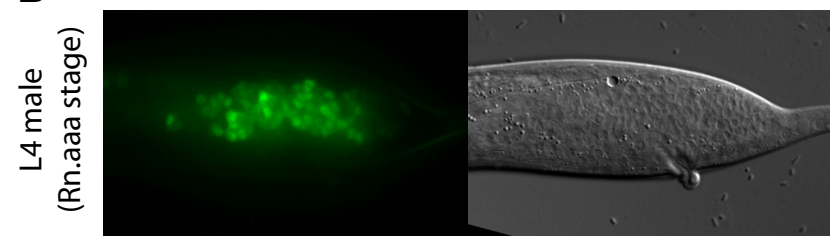

Figure 5. pop-1(RNAi) results in defects in ray cell-fate specification and lin-32 expression. A, A typical adult pop-1(RNAi) male carrying reporters for RnA (tba-9::YFP) and RnB ( $t b b$-6::m $m$ Cherry) fate. Left, Many additional tba-9-expressing cells are apparent (in a wild-type male, $\sim 8-9$ are typically seen) as is a loss of expression of tbb-6. Right, Many rays are missing (bracketed area); in addition, a large fused ray can be seen (arrowhead). B, A typical L4 pop1(RNAi) male. At the Rn.aaa stage, many cells ectopically expressing lin-32::GFP are visible (compare with Fig. 3Avi, a wild-type male at this stage).

also observed large fused rays in many pop-1(RNAi) males (Fig. $5 A$ ). Although these could arise from defects in ray identity specification (Chow and Emmons, 1994; Chow et al., 1995) or in expression of extracellular cues that pattern ray identity (Lints and Emmons, 1999; Lints et al., 2004), they could also represent cell-fate transformations within a single ray sublineage such that multiple cells take on a posterior Rnst-like fate. This would be consistent with recent findings of anterior-to-posterior transformations seen in the seam of pop-1(RNAi) animals (Gleason and Eisenmann, 2010) and supports the idea that pop-1 function is important for both posterior and anterior fates (Mizumoto and Sawa, 2007; Phillips and Kimble, 2009).

\section{The Wnt/ $\beta$-catenin asymmetry pathway regulates lin-32 asymmetry}

We next investigated the role of $\operatorname{lin}$-17 and components of the $\mathrm{Wnt} / \beta$-catenin asymmetry pathway in generating asymmetry in lin-32 expression. In lin-17(n698) males, the initial LIN-32::GFP expression in the Rn.a ray neuroblasts appeared normal (data not shown), consistent with the minor requirement for $l i n-17$ in patterning the seam cell divisions that generate the ray precursor cells (Gleason and Eisenmann, 2010). However, these animals generally failed to show asymmetry of lin-32 expression after Rn.a division, with strong LIN-32::GFP expression detectable in many Rn.aa-Rn.ap pairs in lin-17(n698) males. Asymmetry in the following division was also lost, such that LIN-32::GFP was often detectible in multiple four-celled clusters (Fig. 6A). Expression became undetectable in all ray cells shortly thereafter, indicating that the timing of the inactivation of LIN-32::GFP expression was not affected in these mutants. Similar although less severe effects on LIN-32 asymmetry were observed in lin-17(bx109) and lin44(n1792) mutants (data not shown). We also observed defects in LIN-32::GFP expression in pop-1(RNAi) animals. Many L4 (Rn.aaa stage) animals exhibited large increases in the numbers of cells expressing LIN-32::GFP (Fig. 5B), consistent with a posterior-to-anterior transformation. Some others showed little
A

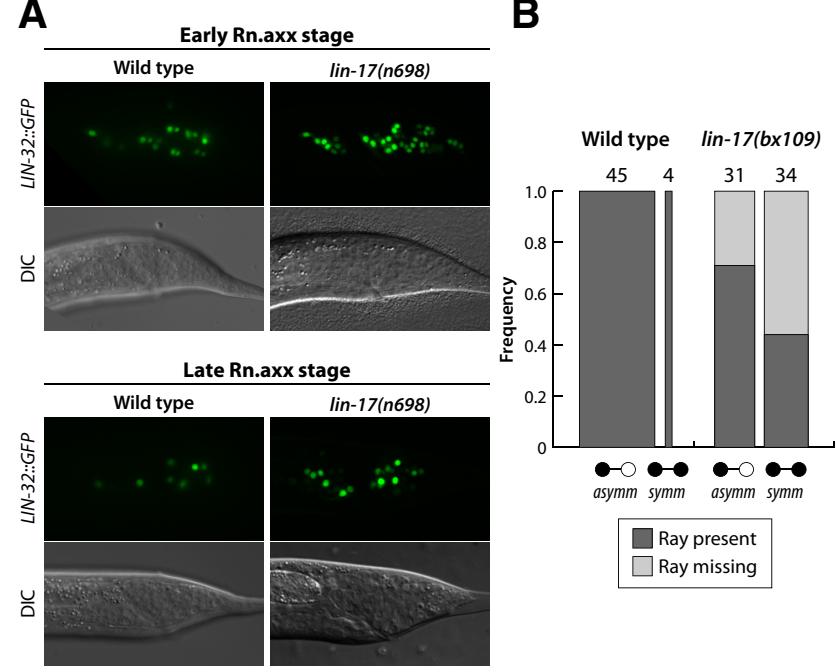

Figure 6. The $\mathrm{Wnt} / \beta$-catenin asymmetry pathway regulates lin-32 expression. $\boldsymbol{A}$, Early (above) and late (below) Rn.axx-stage wild-type and lin-17 mutant L4 males. lin-17(n698) animals harbor extra LIN-32::GFP-positive cells. In early Rn.axx animals, clusters of four strongly GFP-positive cells can be seen, as opposed to the asymmetric pairs seen in wild type. $\boldsymbol{B}, \ln 45$ of 49 wild-type ray lineages examined (in 11 animals), LIN-32::GFP was asymmetrically distributed at the Rn.a[a/p] stage. All of these lineages gave rise to a normal ray. In contrast, only 31 of 65 lineages in lin-17(bx109) males (16 animals) showed LIN-32 asymmetry at the Rn.a[a/p] stage, whereas 34 of 65 appeared to be symmetric. A significantly lower fraction of these symmetric lineages generated rays than did the asymmetric lineages.

or no LIN-32::GFP expression (data not shown). Although this may suggest the reverse transformation, many of these animals had severely disrupted and disorganized tails, such that that the loss of expression could also be secondary to nonspecific necrosis. Together, these findings indicate that the $\mathrm{Wnt} / \beta$-catenin asymmetry pathway controls not only asymmetric cell-fate specification but also asymmetric expression of lin-32 during the ray sublineage.

\section{LIN-32 asymmetry correlates with proper ray development} If Wnt-directed LIN-32 asymmetry is important for ray development, there should be a positive correlation between its asymmetric expression and the development of a ray. To test this, we examined males carrying the weak allele lin-17(bx109), in which some stochastic ray loss is observed but many rays are present. These less severe defects allowed us to reliably identify specific rays by their morphology and position. We first scored the asymmetry of LIN-32::GFP in each of the nine Rn.a[a/p] pairs on a single side of individual L3 males. These animals were then recovered and allowed to develop to adulthood, at which point the number and position of the rays on side of the L3 evaluation was examined (Fig. 6B). Consistent with our hypothesis, we found that $71 \%$ of the lineages in which LIN-32::GFP Rn.a[a/p] asymmetry was normal gave rise to a morphologically normal ray $(n=$ 31 lineages). In contrast, when we observed a lack of normal LIN-32 asymmetry, a ray formed only $44 \%$ of the time $(n=34$ lineages; $p<0.05$, Fisher's exact test). Because LIN-32 asymmetry is a dynamic process and we scored each ray lineage only once, it is likely that our assessment of symmetry is an overestimate (i.e., some lineages scored as "symmetric" may have subsequently gone on to exhibit asymmetry, explaining the relatively large number of rays formed in the symmetric cases). However, even with this potential caveat, our data reveal that LIN-32 asymmetry is a strong predictor of ray development. 

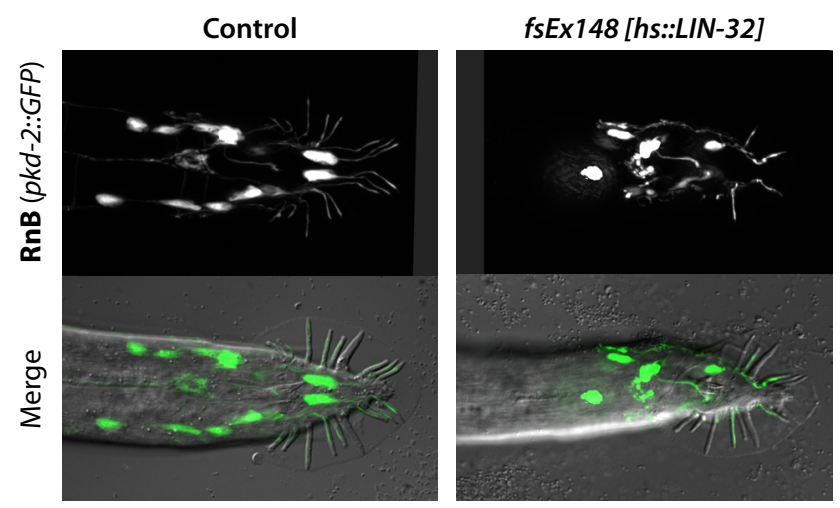

Figure 7. Overriding lin-32 asymmetry disrupts $\mathrm{RnB}$ development. Representative control (nontransgenic) and transgenic hsp::LIN-32::GFP adult males are shown as adults after a transient $\mathrm{L} 3$ heat shock. Control males express the normal complement of $p k d-2::$ GFP-expressing $\mathrm{RnB}$ neurons. In contrast, transgenic males generally have normal rays but exhibit a marked decrease in the extent of $p k d-2::$ GFP expression.

\section{Asymmetric lin-32 expression is necessary for cell-fate specification in the ray sublineage}

Together, our results indicate that the asymmetric expression of LIN-32 could be important for patterning ray cell fates. To test this model, we used a heat-shock-inducible LIN-32::GFP transgene, $f_{s} E x 148$, to ectopically express $l i n-32$ throughout the ray sublineage. We found that a brief heat shock of L3 animals carrying $f_{s} E x 148\left(33^{\circ} \mathrm{C}\right.$ for $\left.20 \mathrm{~min}\right)$ was sufficient to cause LIN-32::GFP expression throughout the tail (data not shown). After recovered animals were allowed to mature to adulthood, we counted rays and examined ray neuron fates in adults with the R[5,7,9]A marker Pdat-1::GFP (egIs1, expressed in R5A, R7A, and R9A) and the RnB marker Ppkd-2::GFP.

We found that ectopic expression of lin-32 only rarely led to ray loss, suggesting that Rnst fate was not disrupted by this manipulation. However, we did often see morphological defects, including fused, stubby, and abnormally positioned rays (data not shown), indicating that Rnst differentiation may be compromised by ectopic lin-32 expression. Examining ray marker expression in these animals, we found that heat-shocked $f_{s} E x 148$ males, but not heat-shocked controls, sometimes possessed one extra Pdat-1::GFP-expressing cell (38\%; $n=34$ sides). Unexpectedly, this extra expression was often in ray 1 or 6 , suggesting an unexpected role for lin-32 in patterning dopaminergic fates between rays. Importantly, we did observe a clear decrease in the number of Ppkd-2::GFP-expressing RnB neurons during ectopic lin-32 expression (Fig. 7). In heat-shocked control males, only 2 of 49 sides scored possessed fewer than the normal complement of eight GFP-positive cells. In contrast, ectopic lin-32 expression lead to complete or partial loss of RnB marker expression in $60 \%$ of 52 sides scored; in this $60 \%$, we observed an average of 5.2 GFP-positive neurons per side. A similar result was obtained using a second marker of RnB fate, Pcwp-4::YFP (data not shown). In contrast, heat shocks delivered during L4 or adulthood led to no defects in ray neuron or Rnst development (data not shown). Although we cannot rule out the possibility that lin-32 overexpression, rather than ectopic expression, underlies these defects in $\mathrm{RnB}$ specification, it seems likely that $\mathrm{RnB}$ defects result from the failure of lin-32 to be downregulated in the posterior branch of the ray sublineage. To further investigate this possibility, we performed heat-shock experiments in lin-32(tm2044) null males. However, this had no significant effects on the limited ray development that occurs in these animals, indicating that the timing and/or expression level of ectopic lin-32 was not sufficient to rescue the early proneural function of $l i n-32$ in the ray neuroblasts (data not shown). We conclude that the forced perdurance of lin-32 activity in posterior branches of the ray sublineage disrupts development of the $\mathrm{RnB}$ neuron, indicating that lin-32 asymmetry is an important component of the cell-fate specification mechanisms operating in the ray sublineage.

\section{Discussion}

The simple developmental architecture of the rays offers a unique developmental system in which to probe the genetic mechanisms that specify neural precursors and allocate specific fates among their progeny. Here, we find that lineage-coupled activity of lin-32 during ray development is mediated by the $\mathrm{Wnt} / \beta$-catenin asymmetry pathway and is important for cell-fate patterning in the ray sublineage. Together with previous results, our findings lead to a model of ray development in which lin-32 links early and late events in cell-fate specification (Fig. 8). In mid-L3, spatial (Chow and Emmons, 1994; Zhao and Emmons, 1995; Salser and Kenyon, 1996; Wrischnik and Kenyon, 1997) and sexual (Shen and Hodgkin, 1988; Ross et al., 2005) patterning mechanisms converge to activate lin-32 in the posterior seam cells, defining the set of ray neuroblasts (Rn.a cells). LIN-32, with its heterodimerization partner HLH-2 (Portman and Emmons, 2000), is thought to activate a set of target genes that endow these cells with the potential to generate neuroglial progeny. The ray neuroblasts then divide to produce the daughters Rn.aa and Rn.ap. Under the influence of the Wnt/ $\beta$-catenin pathway, the Rn.aa cell continues to express lin-32, although its sister does not. This provides molecular evidence that these daughters are not equivalent, indicating that these morphologically indistinguishable intermediate cells have a distinct identity at the level of gene expression (Bertrand and Hobert, 2010). Accordingly, lin-32 may have distinct targets in Rn.aa that allow it to restrict its developmental potential. When Rn.aa divides to produce two postmitotic sisters, lin-32 activity is maintained only in the anterior daughter Rn.aaa. This cell subsequently differentiates into an RnA neuron, whereas its sister undergoes programmed cell death. lin-32 is likely to be important for distinguishing between these two fates (see below), possibly by promoting the expression in Rn.aaa of a transcription factor that specifies RnA-specific characteristics. Thus, these multiple functions of lin-32 and the Wnt/ $\beta$-catenin asymmetry pathway provide a mechanistic link between the earliest event of ray development-specification of the ray neuroblast-and the terminal differentiation of one class of ray neurons.

Because the Wnt/ $\beta$-catenin asymmetry pathway has roles in multiple successive asymmetric divisions in the seam-both before and during ray development (Herman and Horvitz, 1994; Gleason and Eisenmann, 2010)_-disruption of this mechanism leads to a variety of ray phenotypes. However, the most consistent defect observed in lin-44, lin-17, and pop-1(RNAi) males is a gain of RnA marker expression at the expense of RnB marker expression. This result can be explained by two distinct posterior-toanterior transformations of cell fate: transformation of Rn.aap (sister of $\mathrm{RnA}$ ) to an RnA-like fate, or transformation of the precursor Rn.ap to an Rn.aa-like fate, generating an extra RnA neuron and cell fated to die. Thus, repeated posterior-to-anterior transformations in all three ray sublineage divisions should in principle lead to a fourfold increase in the number of RnA neurons. Indeed, we did once observe this phenotype in an egIs1; lin-17(n698) male (data not shown). However, this theoretical maximum is expected to be a rare event, because the probability 


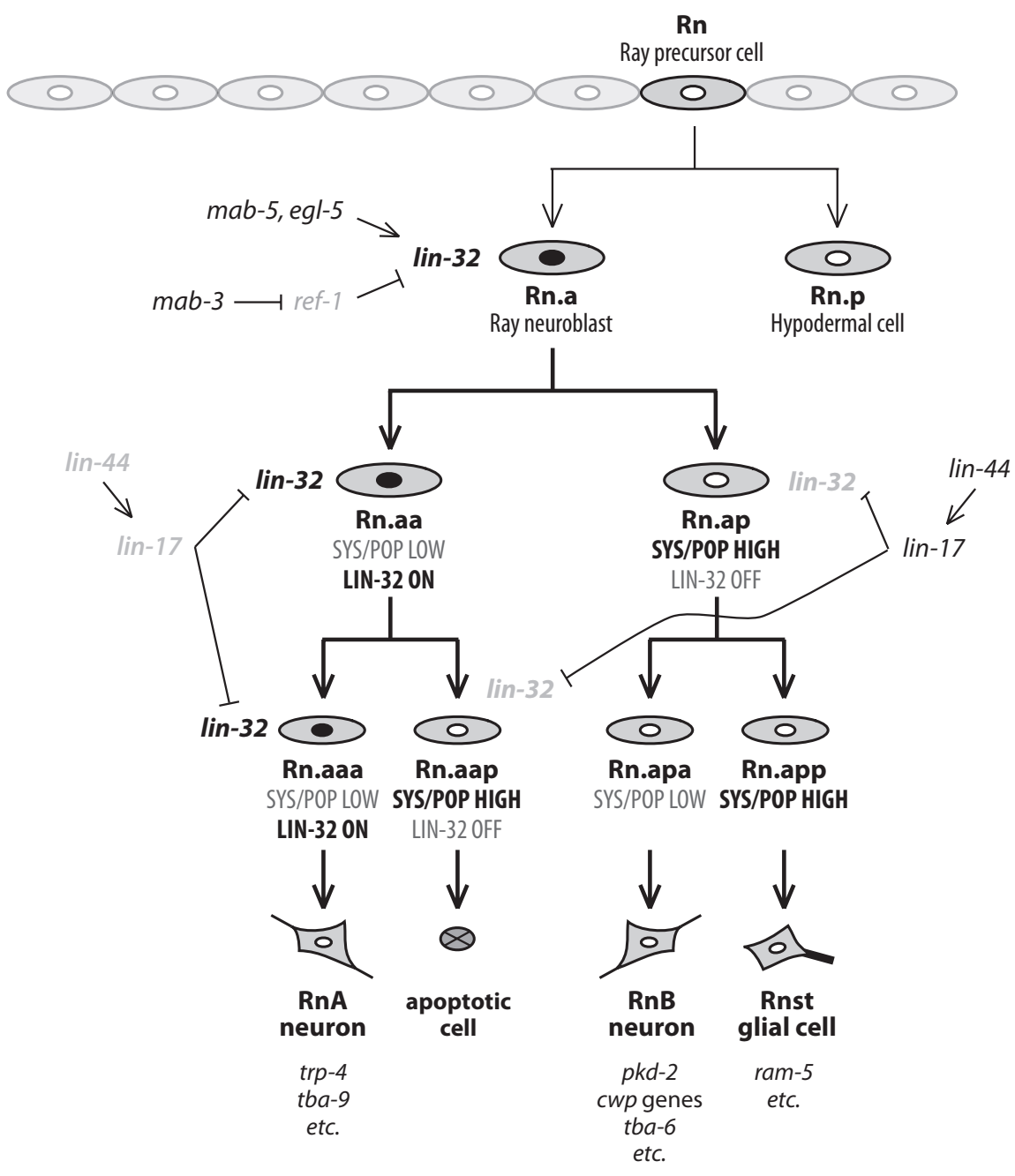

Figure 8. Model: the Wnt/ $\beta$-catenin asymmetry pathway patterns LIN-32 expression during ray development to diversify ray cell fates. Shading of nuclei indicates LIN-32 expression; "SYS/POP" refers to the SYS-1/POP-1 ratio that determines the output of the Wnt/ $\beta$-catenin signaling pathway. In addition to regulation by lin- 17 and lin-44, our findings also indicate a possible role for cell-intrinsic polarity-generating mechanisms. For details, see Discussion.

of each defect in this hypomorphic allele is relatively low and its frequency is balanced by earlier defects that prevent the execution of the entire ray sublineage. Similarly, the modest decrease in the number of $\mathrm{RnB}$ cells is likely to be the net result of early ray sublineage failure, transformation of the Rn.ap intermediate cell to its sister Rn.aa, and possible transformation of the Rn.app ray structural cell to the Rn.apa fate. Together, our results indicate that the $\mathrm{Wnt} / \beta$-catenin asymmetry pathway regulates each cell division in the ray sublineage.

We asked whether LIN-32 asymmetry was important in the patterning of ray cell fates by forcing its expression throughout the ray sublineage. These experiments demonstrated that continued expression of LIN-32 disrupts the differentiation of the $\mathrm{RnB}$, but not RnA, neurons. Because we did not observe a clear transformation of $\mathrm{RnB}$ to $\mathrm{RnA}$ fate, other factors regulated by Wnt signaling but not by lin-32 are likely to be important for promoting RnA differentiation. Interestingly, we did not observe significant loss of the Rnst ray structural cell, suggesting that LIN-32 asymmetry does not have an important role in its specification. Nevertheless, these data indicate that the regulation of LIN-32 by Wnt signaling is critical for the establishment of neural diversity among the progeny of the ray precursor cell.
How does the Wnt/ $\beta$-catenin asymmetry pathway regulate lin-32? During wild-type ray development, LIN-32 expression is lost in the posterior cells of the ray sublineage. Thus, it is unlikely that lin-32 itself is a transcriptional target of the activating SYS-1-POP-1 complex. The absence of clear POP-1 binding sites in the lin-32 promoter is consistent with this idea. Instead, POP-1 may activate a repressor of $l i n-32$ that acts either at the level of LIN-32 transcription or protein stability, possibilities that could be distinguished using a reporter of lin-32 transcriptional activity. It is unlikely that LIN-32 is itself asymmetrically segregated during cell division, because we observe LIN-32::GFP present in both daughter cells immediately after cytokinesis. However, we cannot rule out the possibility that some preferential segregation of LIN-32 contributes to its asymmetry.

\section{lin-32 is not essential for viability or ray development}

An unexpected finding of this work is that lin-32 null males are viable and show strong, but not complete, ray loss. This necessitates some reinterpretation of previous work, in which existing alleles were thought to be hypomorphs. Instead, our analysis of deletion alleles indicates that their ray-loss phenotypes are comparable with those of $e 1926, f_{s} 6$, and $z d 174$, indicating that these latter alleles are also likely to completely remove lin-32 function. In contrast, the $u 282$ allele, a missense change in the basic (DNA-binding) domain of lin-32 (Zhao and Emmons, 1995), causes ray defects stronger than those of the null. One reasonable explanation is that $u 282$ possesses antimorphic (dominant-negative) activity, potentially arising from interference by the LIN-32(u282) protein with the activity of other bHLH factors such as HLH-2, a heterodimerization partner of LIN-32 (Portman and Emmons, 2000). The similarity of phenotypes between lin-32(u282) and hlh-2(bx115); lin-32(tm2044) males is consistent with this possibility, as is the weak semidominance of $u 282$ observed in tra1(e1099); lin-32(u282)/+ pseudomales (Zhao and Emmons, 1995). Nevertheless, the important conclusions of previous studies-that lin-32 is important for triggering execution of the ray sublineage and that lin-32 acts at multiple steps in this lineage (Zhao and Emmons, 1995; Portman and Emmons, 2000)—still stand.

\section{Conserved principles in the diversification of neural fates}

Given the widespread importance of Wnt signaling and atonal-family bHLH transcription factors in patterning the developing metazoan nervous system, our work highlights both shared and disparate principles by which neural cell fates can be patterned. Interestingly, rays use a quite different developmental mechanism than do the sensory bristles in Drosophila to which they have been compared. Although both 
structures are initially specified through the activity of proneural bHLH transcription factors, Drosophila sensory organ precursors rely on the asymmetric segregation of factors such as Numb that bias Notch-mediated specification of cell fates (Gönczy, 2008; Knoblich, 2008). In the rays, however, no role for Notch signaling has been reported. Instead, we find that the $\mathrm{Wnt} / \beta$-catenin asymmetry pathway functions to establish developmental asymmetry and that asymmetric expression of the proneural factor LIN-32 itself is important, although not sufficient, for patterning fates in the terminal divisions of the ray sublineage. Thus, anatomically similar structures can be built by taking advantage of different sorts of extracellular cues as appropriate for a particular developmental context.

To what degree is the role of the $\mathrm{Wnt} / \beta$-catenin asymmetry pathway in regulating neural cell-fate specification conserved? Recent studies in C. elegans have shown that cell-fate specification decisions in the embryonic nervous system are also patterned by this mechanism (Bertrand and Hobert, 2009). Interestingly, mammalian Wnt signaling is critical for regulating the proliferation and differentiation of multiple cell types in the developing CNS (Freese et al., 2010). Similarly, proneuralclass bHLH factors related to atonal and lin-32 have central roles in specifying neural precursors and regulating cell fate (Bertrand et al., 2002). However, the relationships between atonal-family transcription factors and Wnt signaling is not well understood. Notably, several neural structures do, like the rays, use a single proneural-class factor for multiple developmental functions in a single lineage (Guillemot, 2007). It will be interesting to ask whether Wnt-mediated regulation of these factors contributes to the "multitasking" that they perform in these situations.

\section{References}

Banerjee D, Chen X, Lin SY, Slack FJ (2010) kin-19/casein kinase I alpha has dual functions in regulating asymmetric division and terminal differentiation in C. elegans epidermal stem cells. Cell Cycle 9:4748-4765.

Barr MM, Garcia LR (2006) Male behavior. In: Wormbook. E-book available at www.wormbook.org.

Barr MM, DeModena J, Braun D, Nguyen CQ, Hall DH, Sternberg PW (2001) The Caenorhabditis elegans autosomal dominant polycystic kidney disease gene homologs lov-1 and pkd-2 act in the same pathway. Curr Biol 11:1341-1346.

Bertrand N, Castro DS, Guillemot F (2002) Proneural genes and the specification of neural cell types. Nat Rev Neurosci 3:517-530.

Bertrand V, Hobert O (2009) Linking asymmetric cell division to the terminal differentiation program of postmitotic neurons in C. elegans. Dev Cell 16:563-575.

Bertrand V, Hobert O (2010) Lineage programming: navigating through transient regulatory states via binary decisions. Curr Opin Genet Dev 20:362-368.

Brenner S (1974) The genetics of Caenorhabditis elegans. Genetics 77:71-94.

Chalfie M, Au M (1989) Genetic control of differentiation of the Caenorhabditis elegans touch receptor neurons. Science 243:1027-1033.

Chow KL, Emmons SW (1994) HOM-C/Hox genes and four interacting loci determine the morphogenetic properties of single cells in the nematode male tail. Development 120:2579-2592.

Chow KL, Hall DH, Emmons SW (1995) The mab-21 gene of Caenorhabditis elegans encodes a novel protein required for choice of alternate cell fates. Development 121:3615-3626.

Doitsidou M, Flames N, Lee AC, Boyanov A, Hobert O (2008) Automated screening for mutants affecting dopaminergic-neuron specification in $C$. elegans. Nat Methods 5:869-872.

Eisenmann DM (2005) Wnt Signaling. In: Wormbook. E-book available at www.wormbook.org.

Ferreira HB, Zhang Y, Zhao C, Emmons SW (1999) Patterning of Caeno- rhabditis elegans posterior structures by the Abdominal-B homolog, egl- 5 . Dev Biol 207:215-228.

Freese JL, Pino D, Pleasure SJ (2010) Wnt signaling in development and disease. Neurobiol Dis 38:148-153.

Gleason JE, Eisenmann DM (2010) Wnt signaling controls the stem cell-like asymmetric division of the epithelial seam cells during C. elegans larval development. Dev Biol 348:58-66.

Gleason JE, Szyleyko EA, Eisenmann DM (2006) Multiple redundant Wnt signaling components function in two processes during C. elegans vulval development. Dev Biol 298:442-457.

Gönczy P (2008) Mechanisms of asymmetric cell division: flies and worms pave the way. Nat Rev Mol Cell Biol 9:355-366.

Guillemot F (2007) Spatial and temporal specification of neural fates by transcription factor codes. Development 134:3771-3780.

Herman M (2001) C. elegans POP-1/TCF functions in a canonical Wnt pathway that controls cell migration and in a noncanonical Wnt pathway that controls cell polarity. Development 128:581-590.

Herman MA, Horvitz HR (1994) The Caenorhabditis elegans gene lin-44 controls the polarity of asymmetric cell divisions. Development 120:1035-1047.

Hirabayashi Y, Gotoh Y (2010) Epigenetic control of neural precursor cell fate during development. Nat Rev Neurosci 11:377-388.

Hurd DD, Miller RM, Núñez L, Portman DS (2010) Specific alpha- and beta-tubulin isotypes optimize the functions of sensory cilia in Caenorhabditis elegans. Genetics 185:883-896.

Kidd AR 3rd, Miskowski JA, Siegfried KR, Sawa H, Kimble J (2005) A betacatenin identified by functional rather than sequence criteria and its role in Wnt/MAPK signaling. Cell 121:761-772.

Knoblich JA (2008) Mechanisms of asymmetric stem cell division. Cell 132:583-597.

Korswagen HC, Herman MA, Clevers HC (2000) Distinct beta-catenins mediate adhesion and signalling functions in C. elegans. Nature 406:527-532.

Krause M, Park M, Zhang JM, Yuan J, Harfe B, Xu SQ, Greenwald I, Cole M, Paterson B, Fire A (1997) A C. elegans E/Daughterless bHLH protein marks neuronal but not striated muscle development. Development 124:2179-2189.

Lints R, Emmons SW (1999) Patterning of dopaminergic neurotransmitter identity among Caenorhabditis elegans ray sensory neurons by a TGF-beta family signaling pathway and a Hox gene. Development 126:5819-5831.

Lints R, Jia L, Kim K, Li C, Emmons SW (2004) Axial patterning of C. elegans male sensilla identities by selector genes. Dev Biol 269:137-151.

Livesey FJ, Cepko CL (2001) Vertebrate neural cell-fate determination: lessons from the retina. Nat Rev Neurosci 2:109-118.

Mizumoto K, Sawa H (2007) Two betas or not two betas: regulation of asymmetric division by beta-catenin. Trends Cell Biol 17:465-473.

Nass R, Hall DH, Miller DM 3rd, Blakely RD (2002) Neurotoxin-induced degeneration of dopamine neurons in Caenorhabditis elegans. Proc Natl Acad Sci U S A 99:3264-3269.

Phillips BT, Kimble J (2009) A new look at TCF and beta-catenin through the lens of a divergent C. elegans Wnt pathway. Dev Cell 17:27-34.

Phillips BT, Kidd AR 3rd, King R, Hardin J, Kimble J (2007) Reciprocal asymmetry of SYS-1/beta-catenin and POP-1/TCF controls asymmetric divisions in Caenorhabditis elegans. Proc Natl Acad Sci USA 104:3231-3236

Portman DS, Emmons SW (2000) The basic helix-loop-helix transcription factors LIN-32 and HLH-2 function together in multiple steps of a $C$. elegans neuronal sublineage. Development 127:5415-5426.

Portman DS, Emmons SW (2004) Identification of C. elegans sensory ray genes using whole-genome expression profiling. Dev Biol 270:499-512.

Ren H, Zhang H (2010) Wnt signaling controls temporal identities of seam cells in Caenorhabditis elegans. Dev Biol 345:144-155.

Ross JM, Kalis AK, Murphy MW, Zarkower D (2005) The DM domain protein MAB-3 promotes sex-specific neurogenesis in C. elegans by regulating bHLH proteins. Dev Cell 8:881-892.

Salser SJ, Kenyon C (1996) A C. elegans Hox gene switches on, off, on and off again to regulate proliferation, differentiation and morphogenesis. Development 122:1651-1661.

Sawa H, Lobel L, Horvitz HR (1996) The Caenorhabditis elegans gene lin-17, 
which is required for certain asymmetric cell divisions, encodes a putative seven-transmembrane protein similar to the Drosophila frizzled protein. Genes Dev 10:2189-2197.

Shen MM, Hodgkin J (1988) mab-3, a gene required for sex-specific yolk protein expression and a male-specific lineage in C. elegans. Cell 54: 1019-1031.

Siegfried KR, Kimble J (2002) POP-1 controls axis formation during early gonadogenesis in C. elegans. Development 129:443-453.

Sulston JE, Albertson DG, Thomson JN (1980) The Caenorhabditis elegans male: postembryonic development of nongonadal structures. Dev Biol 78:542-576

Teng Y, Girard L, Ferreira HB, Sternberg PW, Emmons SW (2004) Dissec- tion of cis-regulatory elements in the C. elegans Hox gene egl-5 promoter. Dev Biol 276:476-492.

Wrischnik LA, Kenyon CJ (1997) The role of lin-22, a hairy/enhancer of split homolog, in patterning the peripheral nervous system of $C$. elegans. Development 124:2875-2888.

Zhang Y, Emmons SW (1995) Specification of sense-organ identity by a Caenorhabditis elegans Pax-6 homologue. Nature 377:55-59.

Zhao C (1995) Developmental control of peripheral sense organs in $C$. elegans by a transcription factor of the bHLH family. PhD thesis, Albert Einstein College of Medicine.

Zhao C, Emmons SW (1995) A transcription factor controlling development of peripheral sense organs in C. elegans. Nature 373:74-78. 